\title{
RECTIFICACIÓN DEL REGISTRO CIVIL DE PERSONAS TRANSEXUALES: LA VIOLENCIA SIMBÓLICA EN LA TRANSICIÓN DE GÉNERO
}

\author{
Alexandre Botelho ${ }^{1}$ \\ Tamara Rossweiler Marques Cardoso ${ }^{2}$
}

Resumen: La transexualidad es tema de intensos y variados debates. Sin embargo, la sociedad se desarrolla, construye y desmantela a velocidad superior a la de los cambios legislativos y jurídicos, promoviendo situaciones de vulnerabilidad. El presente trabajo se dedica al análisis de las decisiones que niegan el pedido judicial de rectificación del registro civil de nombre $y$ sexo/género, de personas transexuales, o las condicionan a la cirugía de readecuación sexual. Se buscó verificar si ese conjunto de decisiones representa "violencia simbólica" en perjuicio de las personas transexuales, de acuerdo con el sentido atribuido a esa expresión por Pierre Bourdieu. Inicialmente, se abordaron los aspectos generales de la sexualidad humana de acuerdo con una comprensión pluridimensional de constitución del sujeto. Posteriormente, se estudió la transexualidad, la individualización de las personas transgénero y su adecuación al ambiente social y jurídico. Se concluyó la investigación con el análisis de la existencia de violencia simbólica a las personas transexuales en fallos emblemáticos provenientes de diversos Estados de Brasil. Se buscó verificar si los fundamentos que niegan a la persona humana la posibilidad de identificarse legalmente con el género de su esencia reproducen patrones culturales y morales de la sociedad vigente, con miras a reforzar la violencia simbólica de género.

Palabras-clave: género; identidad de género; registro civil; transexualidad; violencia simbólica.
Abstract: Transsexuality is the subject of intense and varied debates. However, society develops, builds and dismantles at a speed superior to that of legislative and legal changes, promoting situations of vulnerability. The present work is dedicated to the analysis of the decisions that deny the judicial request of

\footnotetext{
${ }^{1}$ Doutorando PPGD/UFSC. Professor UNISUL

${ }^{2}$ Possui graduação em Direito pela UNIVALI e graduação em Licenciatura em Química pela UFSC. Cursando Pós-graduação Stricto Sensu (Mestrado). Cursando Graduação Segunda Licenciatura em Pedagogia.
} 
rectification of the civil registry of name and sex/gender, of transsexual people, or they condition them to the surgery of sexual readjustment. We sought to verify if this set of decisions represents "symbolic violence" to the detriment of transgender people, in accordance with the sense attributed to that expression by Pierre Bourdieu. Initially, the general aspects of human sexuality were approached according to a multidimensional understanding of the subject's constitution. Subsequently, transsexuality, the individualization of transgender people and their adaptation to the social and legal environment were studied. The investigation was concluded with the analysis of the existence of symbolic violence to transsexual persons in emblematic faults coming from different States of Brazil. We sought to verify if the foundations that deny the human being the possibility of legally identifying with the genre of their essence reproduce cultural and moral patterns of the current society, with a view to reinforcing the symbolic violence of gender.

Keywords: gender; gender identity; civil registration; transsexuality; symbolic violence.

\section{INTRODUCCIÓN}

La pesquisa pretende investigar la existencia de violencia simbólica, es decir, el proceso de violencia suave, insensible, invisible a sus propias víctimas, que se ejerce esencialmente por las vías puramente simbólicas de la comunicación y del conocimiento o, más precisamente, del desconocimiento, del reconocimiento o, en última instancia, del sentimiento (BOURDIEU, 2011, p. 7-8), en las decisiones judiciales que niegan la solicitud de rectificación de registro civil de personas transexuales o lo condicionan a la realización de una cirugía de reasignación sexual.

La transexualidad es tema de debate en diversas áreas del conocimiento (antropología, filosofía, psicología, psiquiatría, medicina etc.), cada uno con sus respectivos referentes teóricos. No es diferente en el derecho. Las tradiciones de investigación (LAUDAN, 2011) condicionan al investigador a proporcionar respuestas a los problemas que no provocan cambios significativos en el cuerpo social. Los cambios legislativos y jurídicos (plan judicial) ocurren a una velocidad inferior a las acontecidas en la sociedad y oriundas de los más variados movimientos sociales, que buscan la 
equiparación y ampliación de derechos, teniendo como materia prima las construcciones y desconstrucciones de las personas que componen la sociedad.

Con el fin de reducir este hiato entre los anhelos sociales y las respuestas legislativas, el Poder Judicial ejerce, en la práctica y con impacto extraprocesal (erga omnes), verdadera actividad legislativa, ya que, es necesario recordar que, en el marco procedimental brasileño, que prohíbe juzgar el non liquet (no está claro) (artículo 140, Código de Procedimiento Civil/2015²), obligando al juez, al final de cada demanda, a entregar la solución al litigio presentado, independientemente de la existencia o no de legislación pertinente.

A menudo, la brecha generada por la ausencia de legislación, promueve situaciones perjudiciales para los grupos minoritarios, no tutelados por el ordenamiento jurídico. En el ámbito del derecho, las decisiones judiciales se dictan en conformidad con el libre convencimiento motivado de los jueces (artículo 371, CPC/2015), y debidamente fundamentadas (artículo 93, IX, Constitución de la República Federativa del Brasil $^{3}$ ), son válidas y obligan a las partes y terceros.

\footnotetext{
${ }^{2}$ En adelante, también: CPC/2015, CPC.
}

La justificación de las decisiones judiciales a menudo presenta una norma legal particular para los valores que la sociedad toma como bello, justo y bueno, estableciendo el deseo de adaptar al individuo a determinado estándar. El poder es capaz de orientary determinar comportamientos comprendidos como normalizados, aunque no sean normatizados (legislados). El simbolismo contenido en esas decisiones extravasa el texto formal y establece orientaciones de conductas y censuras.

Los transexuales son personas que poseen un sexo/género psicosocial diferente de su sexo biológico y muchas veces necesitan adecuarse al medio en que viven, sea por convicción íntima, sea por necesidad de pertenecer a una comunidad (PICAZIO, 1998, p. 45).

Resulta claro que no todos los transexuales necesitan o quieren equipararse corporalmente al género al que pertenecen realmente y entonces surge el conflicto (deseo versus norma) que puede convertirse en una disputa legal. Algunas decisiones judiciales, que serán analizadas más adelante, entienden que si el transexual se declara no perteneciente al sexo, definido en

\footnotetext{
${ }^{3}$ En adelante, también: CRFB/88, CRFB.
} 
función de los genitales, entonces debe ser adecuado para el género al que pertenece. Así, parece apropiado entender que el modelo judicial predominante, en las Cortes brasileñas, revela la adopción del sistema binario descripto por Scott (1990, p. 17), es decir, la persona debe obligatoriamente ajustarse al género femenino o masculino.

De otra parte, la imposición de un procedimiento quirúrgico de carácter experimental (art. 15, Resolución $\mathrm{n}^{\circ}$. 2.803, de 19 de noviembre de 2013, del Sistema Único de Salud ${ }^{4}$ ) puede ser identificado como la imposición de la violación del cuerpo, la mutilación coercitiva, que revela, en razón de ello, la violencia simbólica que se encuentra en las sutilezas de un sistema jerárquico de poder y reconocimiento (BOURDIEU, 2011, p. 54).

\section{DIVERSIDAD SEXUAL Y GÉNERO}

Diversas teorías buscan explicar las subjetividades humanas en lo que se refiere a la sexualidad (SUTTER, 1993; CHAVES, 1994; COSTA, 1994; PERES, 2011) y aunque ninguna sea unánime, se cree que se complementan. Así, se comprende la sexualidad como una construcción social "contribuyendo ciertamente para que sea uno de los aspectos más conflictivos, controvertidos y desconocidos de nuestra cultura que, a su vez, crea modelos estanque en los que pretende a menudo encajar y clasificar" (SILVEIRA, 2006,p. 23).

Se suele interpretar la sexualidad como algo estandarizado, con reglas rígidas a ser observadas, establecidas por grupos que desean que los demás sigan el mismo patrón sexual, lo que provoca muchos conflictos. La sociedad, por medio de una construcción histórica, busca patrones que normalizan las relaciones humanas y lo que surge como diferente recibe un tratamiento también diferente.

Para el mantenimiento de lo que es aceptado como verdadero, por el simple hecho de ser igual, el camino es no ver o escuchar cualquier cosa que se aparte del comportamiento de la mayoría. [...] Cualquier intento de escapar de los estereotipos estratificadas se identifica como el vicio, el pecado o el crimen y etiquetado inmoral, un atentado a la ética y a las buenas costumbres (DIAS, 2004, p.19, énfasis añadido).

\footnotetext{
${ }^{4}$ En adelante, también: SUS.
} 
El comportamiento que no se presenta como estándar, perteneciendo a la colectividad denominada "normal", es marginado, es decir, puesto al margende la sociedad, precisamente por no existir el sentimiento de pertenencia a lo que es "central" y por el no reconocimiento de los valores emanados por esta fuente "central" de referencia de valores sociales.

Mismo existiendo otros referenciales teóricos que lleven en consideración la fluidez del sujeto, que puede transitar por otros géneros, incluso desde el ángulo de la performance, como indicado por la teoría queer (Butler, 2008), se ha optado por Claudio Picazio (1998) que trata de forma objetiva el conjunto de caracteres básicos de cada sujeto, en la cual el ser humano puede presentar características básicas de sexualidad, que son: sexo biológico, identidad sexual/género, papel sexual y orientación sexual.

El sexo biológico es aquel determinado por las características genotípicas y principalmente fenotípicas presentadas por el individuo $\mathrm{y}$ reconocidas en el momento de su nacimiento. Así, sexo biológico puede ser masculino, femenino $\mathrm{o}$ intersexual/hermafrodita, cuando hay rasgos de ambos sexos o incluso predominio de uno de ellos (se predomina el uso de la expresión intersexual) (PICAZIO, 1998, p. 19-20).

La identidad sexual de género se refiere al auto reconocimiento del individuo, como perteneciente a uno de los géneros masculino $\mathrm{y}$ femenino (PICAZIO, 1998, p. 21). También existe el reconocimiento del género neutro en Australia, que se refiere a aquellas personas que no poseen una identificación con ninguno de los géneros previamente asignados. En Brasil prevalece el binarismo de género (GARCIA, 2015, p. 118).

Picazio utiliza la expresión "identidad sexual" (1998, p. 21), pero concuerda con Robert Stoller, citado por Silveira (2006, p. 28) para quien, en verdad, se trata de "identidad de género", Una vez que retira el carácter sexualizador de la identidad (SARTORI, 2006, p. 10). La identidad de género trata de la convicción que el individuo tiene de su propia identidad, un sentimiento interno que conduce a comportamientos referentes a ese género y no al correspondiente a los genitales (PICAZIO, 1998, p. 21).

Los papeles sexuales difieren según la región, cultura o momentos 
históricos analizados y muchas veces son determinados por el patrón social vigente en la época. Algunos papeles sexuales se encuentran internalizados en la cultura, sin embargo conduce al cuestionamiento relativo a la orientación sexual. El estándar social lleva a la formación de estereotipos estructurales del individuo, promoviendo conclusiones que no siempre coinciden con la realidad. La orientación sexual aborda el deseo afectivo sexual, el direccionamiento de la libido y tradicionalmente puede ser: heterosexual, homosexual o bisexual (PICAZIO, 1998, p. 24).

La homosexualidad implica dos términos que sirven para identificar y empoderar ${ }^{5}$ los sujetos: los gay y las lesbianas. Estas son aquellas mujeres (por los genitales o por el género) que se relacionan con otras mujeres (definidas por los genitales o por el género). Los gay son aquellos hombres (definidos por los genitales o por el género) que se relacionan con otros hombres (definidos por los genitales o por el género) (PICAZIO, 1998, p. 30).

Se trata de la construcción de concepto de género por la inclinación

\footnotetext{
5 Neologismo. Significa una acción colectiva desarrollada por los individuos cuando participan en espacios privilegiados de decisiones, de conciencia social de los derechos sociales. Esta
}

social, considerando no sólo el sexo, sino también los aspectos políticos, sociales, de clase y la relación de estos parámetros con el poder, efectivamente ejercido o simbólico, presente en las relaciones desiguales y en la subordinación de las mujeres (WOLFF, 2015, p. 95).

El género es analizado como producto de una construcción social relacional entre los sexos (BOURDIEU, 2011, p.32), basado en las diferencias de poder y en la asignación previa de papeles sexuales. A pesar de ello, no se puede confundir el género y/o limitarlo al sexo biológico, aunque sea "una categoría social impuesta sobre un cuerpo sexuado" (SCOTT, 1990, p. 7), la vivencia subjetiva personal y política forma parte de la constitución de los sujetos y la discusión de la propia construcción del género y de la identidad no se limita a los genitales. En este sentido, vale mencionar un fragmento de decisión del Tribunal de Justicia de Santa Catarina:

Cuando se editó la ley de obligatoriedad del registro civil, la distinción entre los dos sexos se hacía fundamentada en la conformación de los genitales. Sin embargo, con el avance del desarrollo científico

conciencia sobrepasa la toma de iniciativa individual de conocimiento y superación de una realidad en la que se encuentra. 
y tecnológico, hay varias otras designaciones de sexo, por lo que la definición de género no puede limitarse sólo al sexo aparente (énfasis añadido).

El reconocimiento de la identidad de género es necesario para la comprensión de las diferencias, pues cada individuo lleva un conjunto de valores constituidos de acuerdo al medio social, enlaces familiares, y las experiencias (in)exitosas de vida, el reconocimiento o no de la sociedad y también de todo el ordenamiento jurídico, visto que éste es un normatizador de relaciones sociales.

\section{TRANSEXUALIDAD}

La base de la comprensión de la transexualidad, como identidad de género es entenderla como carácter independiente y harmónico en el conjunto de las demás características que pueden explicar la sexualidad humana. No se desconoce la discusión sobre los vocablos "transexualismo" (CID-10, F64 - trastorno de identidad sexual, F64.0 transexualidad) y "transexualidad", centralizada principalmente en los conceptos de las áreas de la salud, como medicina, psicología, psiquiatría, entre otros (CARDOSO, 2009, p. 85). Se dice que el sufijo "ismo" tiene sentido de calidad, estado, pero que proporciona una connotación, también, de enfermedad, mientras que el sufijo "dade" también representa calidad, estado, sin implicar una connotación enfermiza (SANTANA, 2007, p. 19; CARDOSO, 2015, p. 13).

Por ser parte de la construcción social y de la identidad de la persona, en cada época, las características femeninas o masculinas se modifican con el paso del tiempo y con las luchas sociales, no se pudiendo decir que este o aquel comportamiento es exclusivo de uno de los géneros. Así pues, no hay porque pensar en características naturalmente femeninas, porque ser mujer es una construcción (LISBÔA, 1998, p. 135).

Hablar de transexualidad implicala reflexión sobre lo que es la sexualidad más allá de las concepciones biológicas, obligándose a pensar en las experiencias de la sexualidad en las esferas pública y privada, tanto con la práctica individual, como práctica social y política (AVILA E GROSSI, 2010, p. 1) (énfasis añadido).

Hablar en transexualidad es deconstruir la sexualidad humana, pues la transitoriedad de los géneros demuestra las desconstrucciones y 
construcciones de los sujetos. La construcción de la identidad mujer y hombre se refiere a las categorías sociales, en cuanto macho/hembra y reproducción, se refiere a las categorías biológicas, no debe haber confusión de conceptos, con miras a la no estandarización de los sujetos (ROUGHGARDEN, 2005, p.25).

\section{VIOLENCIA SIMBÓLICA Y} RETIFICACIÓN DEL REGISTRO CIVIL

Bourdieu resalta que es necesario tener en cuenta la existencia de un "universo social relativamente independiente en relación a las presiones externas, dentro del cual se produce y se ejerce la autoridad jurídica, forma por excelencia de la violencia simbólica legítima cuyo monopolio pertenece al Estado" (p. 211).

Con el fin de identificar la presencia de la violencia simbólica en las decisiones judiciales de rectificación de registro civil, se pasa a analizar algunos juzgados emblemáticos de distintas regiones geopolíticas de Brasil.

Por esa senda, del Tribunal de Justicia de Minas Gerais (2009), se cosecha un trecho de fallo, que a pesar de vencido, merece destaque: "la cirugía sólo tuvo la función de dar apariencia femenina al apelante, pero no lo transformó en mujer en el sentido de la palabra, ya no lo dotó de útero, ovarios y otras características propias y peculiares de las mujeres" (énfasis añadido).

Esta afirmación destaca la necesidad de la discusión iniciada después de la Segunda Guerra Mundial y fortalecida a partir de los años 1960, por la "segunda ola" del feminismo, en la que se discutía justamente el derecho al cuerpo, el establecimiento de carácter reproductivo de la mujer, asignación de función sexual y demarcación de estereotipos (WOLFF, 2015, p. 95).

Para Costa mencionado por Caniato (2008, p. 24), la violencia simbólica "se encarga de capturar el mundo interno de los sujetos para sustituirlo por la internalización de formas de ser, desear, sentir, pensar y actuar queinteresan al mantenimiento de la sociedad". Justamente en este espacio se pueden localizar las decisiones judiciales, que niegan la solicitud de rectificación de registro civil de personas transexuales, o lo condicionan a la cirugía de rediseño sexual, pues la violencia simbólica es sutil, enmascarada, disfrazada y, por lotanto, 
bastante eficaz. Se puede inculcar, sugestionar, censurar, modelar, por medio de una fuerza simbólica, hipnótica, que por sí solo configura la violencia simbólica (BOURDIEU, 2011, p. 54).

Al establecer el lugar adecuado para personas transexuales, se está disfrazando la imposición del poder, de la sumisión, la orientación hacia la correcta, bella y buena posición que la mujer deba ocupar en la sociedad. Al imponer la biologización del ser humano, se está camuflando el poder efectivo por medio de un poder simbólico, que resulta en violencia simbólica de género, comprendida como:

[...] todas las formas de agresión o restricción física, moral, psicológico, emocional, institucional, cultural o patrimonial, que se basa en la organización social de los sexos ya ser llevado contra determinadas personas, explícita o implícitamente, a causa de su estado como el sexo o la orientación sexual (Sardenberg, 2011, p. xx) (énfasis añadido).

El derecho también es campo de producción de violencia simbólica, pues "construye e impone una determinada definición del mundo como legítima, un sentido, un fundamento $\mathrm{y}$ una significación, pero también una dirección y una lógica, una racionalidad concreta" (AZEVEDO, 2011, p. 35).

Aunque se considere una Corte pionera en decisiones que abarquen la población LGBTI, también del Tribunal de Justicia Rio Grande del Sur (2011) se puede destacar un juzgado:

[...] no a previsión, aún, para poner realización de cirugía de modificación de sus órganos sexuales, ni siquiera de recomendación de eventual procedimiento quirúrgico, consta noticia en el expediente que se sometió apenas a la retirada de los órganos reproductores femeninos. Pero: aunque tiene apariencia masculina, $[. .$.$] no existen$ explicaciones detalladas y concretas sobre la anatomía de sus órganos sexuales.[...] Por último, hago hincapié en que la mantención del sexo femenino en la partida de nacimiento no causará situaciones embarazosas para el apelante. (énfasis añadido).

Así, una vez más el magistrado acaba ejerciendo una actividad "formalizadora y formalizante" (AZEVEDO, 2011, p 37), atribuyendo al sujeto su biologización, dejando de considerar su constitución como construcción social. Al considerar que no existe situación embarazosa para aquella persona que se identifica como hombre (hombre trans), pero que permanecerá como mujer (mujer cis) en los 
documentos, es no comprender, mínimamente, la real situación vivenciada, desconocer la constitución pluridimensional de los sujetos y, sí, marcar la violencia simbólica por medio de una sutil información de inexistencia de daños.

Del Tribunal de Justicia de Paraíba (2011) se destaca este posicionamiento:

Entiendo que solamente hay que reconocer la posibilidad de cambio del nombre y el sexo del autor, con el fin de adecuarlo a su realidad, después de la cirugía de reasignación de sexo, ya que el órgano sexual, también, está protegido como una señal identificadora de la personal, en relación al mundo exterior, en la sociedad y en el mundo jurídico (énfasis añadido).

Así también lo hace el Tribunal de Justicia de Mato Grueso (2014), cuando afirma que "Si la parte no se sometió a cirugía de cambio del sexo, no se justifica la solicitud de alteración del sexo y nombre para el femenino en su registro civil".

Por último, probablemente la fundamentación más representativa de la violencia simbólica, se extrae de fallo dictado en 1997 por el Tribunal de Justicia de Río de Janeiro:

$\mathrm{Si}$ el solicitante ostenta apariencia [...] incompatible con su condición [...]tendráque asumir las consecuencias, porque la opción fue de él.[...] Quien nace hombre o mujer, muere como nasció. Genital similar no es auténtico. Auténtico es el hombre ser del sexo masculino y la mujer del sexo femenino, a lo que todo evidencia. (énfasis añadido).

El Tribunal de Justicia de Río de Janeiro no reconoció la identidad de género de una persona transexual, única y exclusivamente por la existencia de genitales asociados, previamente, a un género que no armoniza con aquel realmente vivido. No permitir que el ser humano sea quien realmente cree ser es una violencia simbólica y retrata toda la reproducción de creencias y valores de una sociedad dominante.

\section{CONSIDERACIONES FINALES}

El análisis de los juzgados seleccionados permite afirmar la existencia de violencia simbólica en la motivación de las decisiones judiciales. A pesar de estar debidamente justificadas, las decisiones presentaron las características correspondientes a la violencia simbólica, ya que exhiben el establecimiento de estándares para todos los sujetos de derechos y deberes.

Considerando que las decisiones judiciales formulan conceptos estandarizados de la sociedad, la 
imposición de conductas lleva, inexorablemente, a la exclusión, marginación y al no reconocimiento de las singularidades.

Como sostiene Bourdieu, diversos intereses están involucrados, habiendo una disputa "simbólica" entre el campo jurídico y las diversas áreas del conocimiento, en virtud de la existencia de un poder simbólico, que oprime y rige conductas, proveniente de construcciones históricas basadas en el patriarcado y que, efectivamente, ocasionan la violencia simbólica a aquellos sujetos que están al margen de los estándares exigidos por la sociedad actual.

Por eso Bourdieu considera la existencia de una delegación de autoridad, oriunda del arbitrario cultural y de la reproducción social, sostenidos por las clases dominantes, que impone un orden jurídico sobre el supuesto desorden social.

\section{REFERENCIAS}

ÁVILA, Simone; GROSSI, Miriam Pillar. Maria, Maria João, João: reflexões sobre a transexperiência masculina. In: FAZENDO GÊNERO 9: DIÁSPORAS, DIVERSIDADES, ESLOCAMENTOS, 23 a 26 ago. 201, Florianópolis. iódico do Núcleo de Estudos e Pesquisas sobre Gênero e Direito

Centro de Ciências Jurídicas - Universidade Federal da Paraíba V. 6 - No 03 - Ano 2017 (Spanish Edition) 168 em: <http://www.fazendogenero.ufsc.br/9/re sources/anais/1278255349_ARQUIVO_ Maria,MariaJoao,Joao040721010.pdf>. Acesso em 8 mar. 2015.

AZEVEDO, Rodrigo Ghiringhelli de. A força do direito e a violência das formas jurídicas. Rev. Sociol. Polit. Curitiba, v. 19, n. 40, p. 27-41, out. 2011. Disponível em:

$<$ http://www.scielo.br/scielo.php?script $=$ sci_arttext\&pid=S0104$44782011000300004 \& \operatorname{lng}=$ pt\&nrm=iso >. Acesso em 18 nov. 2015.

BOURDIEU, Pierre e PASSERON, Jean Claude. A reprodução: elementos para uma teoria do sistema de ensino. Rio de Janeiro: Livraria Francisco Alves Editora, 1975.

BOURDIEU, Pierre. A dominação masculina. 10. ed. Tradução de Maria Helena Kühner. Rio de Janeiro: Bertrand Brasil, 2011.

BOURDIEU, Pierre. O poder simbólico. 8. ed. Tradução Fernando Tomaz. Rio de Janeiro: Bertrand Brasil, 2005. 
BRASIL. Código de processo civil. Lei

13.105, de 16 de março de 2015.

Disponível

em:

<http://www.planalto.gov.br/ccivil_03/_ Ato2015-2018/2015/Lei/L13105.htm>.

Acesso em 21 nov. 2015.

BRASIL. Código de processo civil. Lei

5.869, de 11 de janeiro de 1973.

Disponível

em:

<http://www.planalto.gov.br/ccivil_03/1

eis/L5869compilada.htm>. Acesso em

21 nov. 2015.

BRASIL. Constituição da República

Federativa do Brasil de 1988.

Disponível

em:

<http://www.planalto.gov.br/ccivil_03/

Constituicao/ConstituicaoCompilado.ht m>. Acesso em 21 nov. 2015.

BRASIL. Portaria n. 2.803, de 19 de novembro de 2013. Brasília, DF: Ministério da Saúde, 2013. Disponível em:

<http://bvsms.saude.gov.br/bvs/saudele gis/gm/2013/prt2803_19_11_2013.html

>. Acesso em 6 mar. 2015.

BUTLER, Judith. Problemas de gênero: feminismo e subversão da identidade. 2. ed. Tradução de Renato
Aguiar. Rio de Janeiro: Civilização

Brasileira, 2008.

CANIATO, Angela Maria Pires.

Violências e subjetividades: o indivíduo contemporâneo. Psicol. Soc. Porto Alegre, v. 20, n. 1, p. 16-32, abr. 2008 . Disponível em: $<$ http://www.scielo.br/scielo.php?script =sci_arttext\&pid=S0102$71822008000100003 \& \operatorname{lng}=$ pt\&nrm=iso >. Acesso em 18 nov. 2015.

CARDOSO, Tamara Rossweiler Marques. A exigência de cirurgia de transgenitalização para a retificação de registro civil de transexuais masculinos (trans-homens). 2015. $73 \mathrm{f}$. Monografia apresentada ao final do curso de Pós-graduação Lato Sensu em Direito processual Civil. Faculdade de Direito Professor Damásio de Jesus, Florianópolis/SC.

O tratamento dispensado aos transexuais pelos tribunais brasileiros. 2009. 238 f. Monografia apresentada ao final do curso de graduação em Direito. Universidade do Vale do Itajaí, Tijucas/SC. 


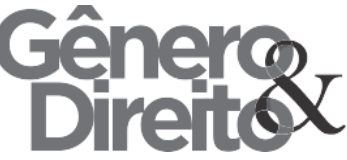

CHAVES, Antônio. Direito à vida e ao

próprio corpo: intersexualidade, transexualidade, transplantes. 2. ed. rev.

e ampl. São Paulo: Revista dos Tribunais, 1994.

COSTA, Ronaldo Pamplona da. Os onze sexos: as múltiplas faces da sexualidade humana. São Paulo: Ed. Gente, 1994.

DIAS, Maria Berenice. Conversando sobre homoafetividade. Porto Alegre: Livraria do Advogado, 2004.

LAUDAN, Larry. O progresso e seus problemas: rumo a uma teoria do crescimento científico. Tradução de Roberto Leal Ferreira. São Paulo: EdUnesp, 2011.

LISBÔA, Maria Regina Azevedo. Masculinidades: as críticas ao modelo dominante e seus impasses. In: GROSSI, Miriam Pillar. PEDRO, Joana Maria (Org.). Masculino, feminino, plural: gênero na interdisciplinaridade. Florianópolis: Ed. Mulheres, 1998.

MINAS GERAIS (Estado). Tribunal de Justiça de Minas Gerais. Apelação Cível n. 1.0024.05.778220-3/001. Apelante: E.P.S. Rel. Des. Edivaldo eriódico do Núcleo de Estudos e Pesquisas sobre Gênero e Direito

Centro de Ciências Jurídicas - Universidade Federal da Paraíba V. 6 - No 03 - Ano 2017 (Spanish Edition)

George dos Santos. $7^{\text {a }}$ Câmara Cível. Julgado em: 6 mar. 2009. Publicado em 7 abr. 2009.

PARAÍBA (Estado). Tribunal de Justiça da Paraíba. Apelação Cível n. 00120090123991001. Relator Genésio Gomes Pereira Filho. Terceira Câmara Cível. Apelante: Rogério Cavalcanti Silva. Apelado: A Justiça Pública. Julgado em: 05 mar. 2011. Publicadoem 7 abr. 2011.

PERES, Ana Paula Ariston Barion. Transexualismo: o direito a uma nova identidade sexual. Rio de Janeiro: Renovar, 2011.

PESQUISA. Transexualismo.

MedicinaNet. Disponível em: <http://www.medicinanet.com.br/cid10/ 1554/f64_transtornos_da_identidade_se xual.htm>. Acesso em 20 set. 2014.

PICAZIO, Claudio. Sexo secreto: temas polêmicos da sexualidade. São Paulo: Hummus, 1998.

RIO DE JANEIRO (Estado). Tribunal de Justiça do Rio de Janeiro. Apelação Cível n. 1993.001.06617. Relator: Desembargador Geraldo Batista. Oitava 
Câmara Cível. Julgado em: 18 mar.

1997. In: RIOS, Roger Raupp. (Org.).

Em defesa dos direitos sexuais. Porto

Alegre: Livraria do Advogado, 2007.

RIO GRANDE DO SUL (Estado).

Tribunal de Justiça do Rio Grande do

Sul. Apelação Cível n. 70042797167.

Relator: Desembargador André Luiz

Planella Villarinho. Sétima Câmara

Cível. Julgado em 14 dez. 2011.

ROUGHGARDEN, Joan. Evolução do gênero e da sexualidade. Tradução de Maria Edna Tenório Nunes. Londrina:

Editora Planta, 2005.

SANTA CATARINA (Estado).

Tribunal de Justiça de Santa

Catarina. Apelação Cível n.

2011.034720-1. Comarca de Rio do Sul, Apelante: Ministério Público de Santa

Catarina. Apelado: S. J. (L.C.J). Terceira

Câmara Cível. Relator: Desembargador Saul Steil, Julgado em 23 ago. 2011.

SANTANA, Karine Cristina.

Homossexualidade: percepção de universitários. 2007. 66 f. Monografia (apresentada ao final do curso de Psicologia). Universidade do Vale do Itajaí.
SARDENBERG, Cecilia M. B. A violência simbólica de gênero e a lei "antibaixaria" na Bahia. OBSERVEObservatório de Monitoramento da Lei Maria da Penha. NEIM/UFBA. Disponível em: <www.observe.ufba.br/noticias/exibir/3 44>. Acesso em 08 nov. 2015.

SARTORI, Ari José. BRITTO, Néli

Suzana. (Org.) Gênero na educação: espaço para a diversidade. 2. ed. Florianópolis: Genus, 2006.

SCOTT, Joan. Gênero: uma categoria útil para análise histórica. Educação e sociedade, Porto Alegre, v. 16, n. 2, p. 522, Jul./Dez 1990. Tradução de Christine Rufino Dabat e Maria Betânia Ávila. Disponível em: $<$ http://disciplinas.stoa.usp.br/pluginfile. php/185058/mod_resource/content/2/G \%C3\%AAnero-Joan\%20Scott.pdf>. Acesso em 10 out. 2015.

SILVEIRA, Esalba Maria Carvalho. De tudo fica um pouco: a construçãosocial da identidade do transexual. 2006. $304 \mathrm{f}$. Tese (Doutorado em Serviço Social) Faculdade de Serviço Social, Pontifícia Universidade Católica do Rio Grande do 
Sul, Porto Alegre, 2006. Disponível em:

$<$ http://tede.pucrs.br/tde_busca/arquivo.

php?codArquivo $=18>$. Acesso em 21

nov. 2014.

SUTTER, Matilde Josefina.

Determinação e mudança de sexo:

aspectos médicos legais. São Paulo:

Revista dos Tribunais, 1993.

WOLFF, Cristina Scheibe. Gênero: um

conceito importante para o

conhecimento do mundo social. In:

GROSSI, Miriam Pillar; GARCIA, Olga

Regina Zigelli; LOZANO, Marie-Anne;

MAGRINI, Pedro Rosas (Org.).

Especialização em gênero e

diversidade na escola, modalidade a

distância. Florianópolis: UFSC, 2015. 